doi: $10.15407 /$ ujpe62.06.0481

V.N. STARKOV, A.A. BORSHCH, I.S. GANDZHA, P.M. TOMCHUK

Institute of Physics, Nat. Acad. of Sci. of Ukraine

(46, Prosp. Nauky, Kyiv 03028, Ukraine; e-mail: starkov@iop.kiev.ua)

\title{
SOME EXAMPLES OF SEEMINGLY PLAUSIBLE PACS 06.20.Dk, 42.30.-d INTERPRETATION OF EXPERIMENTAL RESULTS
}

\begin{abstract}
Some examples of a seemingly plausible but wrong interpretation of experimental results have been considered on the basis of the theory of rigorous mathematical interpretation of experimental data. The ability of apparatus errors to significantly affect the measurement results in optical experiments is demonstrated. Ignoring this fact can result in erroneous conclusions concerning the physical nature of the optical phenomena under consideration.

Ke ywords: optical experiment, apparatus errors, ill-posed problems, regularization method.
\end{abstract}

\section{Introduction}

The fact that the reliability of interpretation of the results of physical studies is of fundamental importance has been emphasized by different outstanding scientists $[1,2]$. In particular, O.S. Davydov wrote [2]: "Any scientific research in physics (and not only in physics) is undoubtedly associated with the interpretation of the results obtained. This interpretation is often called the 'elucidation of the physical sense' or the reaching of 'apprehension' of the studied phenomena. As a rule, the interpretation of a physical phenomenon reflects the level of science development in the corresponding period. Therefore, it is not absolute, but can change in time". A correct interpretation of the results of a scientific physical experiment inevitably demands that the researcher should have a clear concept about the interaction between all components of the experimental process. This understanding, in turn, cannot be complete and reliable, if modern achievements of theoretical, mathematical, and computational physics are ignored.

A very important qualitative circumstance of this work consists in the consideration of the fact that ex-

(C) V.N. STARKOV, A.A. BORSHCH, I.S. GANDZHA, P.M. TOMCHUK, 2017

ISSN 2071-0194. Ukr. J. Phys. 2017. Vol. 62, No. 6 perimental results include measurement errors, since any experiment is always carried out on a real experimental installation. This set of data together with errors of various kinds forms a basis, on which serious difficulties arise quite often. In this work, we examine a few examples of experimental data, for which their interpretation seems plausible, but is wrong.

Section 2 is devoted to the description of basic mathematical concepts and methods applied to solve the problems of interpretation of experimental data. The rigorous mathematical formulation of the latter can often be reduced to the solution of the so-called ill-posed problems [3-5]. Practical needs to analyze the results of experimental researches in physics, geophysics, biophysics, ecology, and many other scientific domains are so urgent that the task to critically examine the modifications and developments of the methods applied to the solution of ill-posed problems still remains challenging. In spite of a considerable number of fundamental books, reviews, and scientific articles, the body of publications dealing with the problems of the numerical solution of ill-posed problems continues to grow. Among those publications, a series of works by Academician of the Russian Academy of Sciences V.N. Strakhov [6-16] can be distinguished by his unordinary approach to the A.N.Ti- 
khonov regularization method. In those works, an attempt was made to prove that the classical regularization method for the solution of systems of linear algebraic equations with approximately given right-hand sides does not satisfy the requirements of geophysical practice, being "defective".

In Section 3, on the basis of expounded mathematical methodology, a possible plausible interpretation of the availability of experimental error in applied researches is analyzed. This situation arises quite often, e.g., when considering the results of I-scan (from "intensity scanning") optical experiment. Here, in contrast to the Z-scan experiment, where the specimen is moved along the axis $O z$ through the focus of Gaussian beam, the intensity of the laser beam incident on the specimen is varied in wide limits [17-22]. In this case, the account of hardware features inherent to the measurement installation is extremely important, especially at low-intensity sections where instrument errors play a crucial role in the measurement results. A seemingly plausible interpretation of such results can give rise to wrong conclusions about the orders of magnitudes for susceptibilities in various media. In this work, we discuss an example of experiment in which a danger of such an interpretation can emerge. This part of the paper has an applied value for the interpretation of experimental data, being presented by us for the first time.

Conclusions and final remarks are made in Section 4.

\section{Mathematical Basis of Interpretation of the Results of V.N. Strakhov's Hypothesis}

Let us consider a system of linear algebraic equations (SLAE)

$A x=u_{\delta}=u+\delta u$,

where $u \in E$ and $\delta u \in E$ are the vectors of useful signal and its error, respectively ( $E$ is a linear space, and $\operatorname{dim} E=N) ; A$ is a given $N \times M$-matrix; and $x \in R$ is a sought $M$-vector ( $R$ is a linear space, and $\operatorname{dim} R=M)$.

For the solution of SLAE (2.1), V.N. Strakhov has proposed a new theory of regularization, which was based on two hypotheses [7-9, 11, 14, 15]. Namely,

1) the error vector satisfies the following inequalities:

$0 \leqslant \inf _{x \in R}\left\|u_{\delta}-A x\right\|_{E}^{2}<\delta_{\min }^{2} \leqslant$

$\leqslant\|\delta u\|_{E}^{2} \leqslant \delta_{\max }^{2}<+\infty$; where $\|\delta u\|_{E}$ is the length of vector $\delta u\left(\|\delta u\|_{E} \in\right.$ $\left.\left[\delta_{\min }, \delta_{\max }\right]\right)$;

2 ) the vectors $u$ and $\delta u$ are mutually orthogonal, i.e.

$(u, \delta u)=0$.

For instance, in work [6], we read: "It is evident that the vectors $u$ and $\delta u$ in the initial system (2.1) are coupled by no analytical relation in the general case; furthermore, in many practically important cases, the a priori condition $(u, \delta u)=0$ can be adopted. Hence, if a functional relation appears between the vectors $\tilde{u}_{\alpha}$ and $\Delta u_{\alpha}$, which are estimates of the vectors $u$ and $\delta u$, in the framework of any regularization construction, the construction of this sort has to be regarded as defective (pathological). The same statement is evidently valid in the case where a functional relation between the vectors $x_{\alpha}$ and $r_{\alpha}=u_{\delta}-A x_{\alpha}$ emerges within the limits of any regularization construction.

Now, let us demonstrate that ... the general construction of additive regularization is defective; hence, the Lavrent'yev, Tikhonov ... constructions turn out to be defective".

V.N. Strakhov used the a priori assumption that Eq. (2.3) is obeyed even before the publication of work [6] (see, e.g., works $[13,16])$.

Strakhov's works did not contain any assumption concerning the magnitude of the scalar product $\left(u_{\delta}, \delta u\right)$. In other words, in a lot of practically important cases, this quantity can be positive, negative, and sometimes equal to zero. This statement follows from the fact that, in the general case, the vectors $u$ and $\delta u$ are not coupled by any analytical relation, leaving aside the definition

$u_{\delta}=u+\delta u$

which follows from Eq. (2.1). However, this is enough to draw some conclusions. Let us be convinced of that. Let us scalarly multiply relation (2.4) by the vector $\delta u$,

$\left(u_{\delta}, \delta u\right)=(u, \delta u)+\|\delta u\|_{E}^{2}$.

Using hypothesis (2.3) and assuming that the product $\left(u_{\delta}, \delta u\right)$ is negative, we may obtain from Eq. (2.5) that

$0 \geqslant\left(u_{\delta}, \delta u\right)=\|\delta u\|_{E}^{2}$.

ISSN 2071-0194. Ukr. J. Phys. 2017. Vol. 62, No. 6 
However, the inequality $\|\delta u\|_{E}^{2}<0$ is absurd by its sense, whereas the equality $\|\delta u\|_{E}^{2}=0$ contradicts condition (2.2). Therefore, only the variant where

$\left(u_{\delta}, \delta u\right)=\|\delta u\|_{E}^{2}>0$

remains. This relation is undoubtedly satisfied, e.g., if $u_{\delta}=\delta u$, i.e. when the experimental results are wrong.

If one also takes into account that relation (2.3) is valid at $\delta u=0$, i.e. in the trivial case, the examined situation can be described more exactly as follows. Let the vectors $\delta u$ be elements of the space $R^{N_{\delta}}$ $\left(1 \leqslant N_{\delta} \leqslant N\right)$, and let the vectors $u$ be elements of the space $R^{N_{u}}\left(N_{u}+N_{\delta}=N\right)$. In addition, the space $R^{N}$ is a direct sum of the subspaces $R^{N_{u}}$ and $R^{N_{\delta}}$, i.e.

$R^{N}=R^{N_{u}} \oplus R^{N_{\delta}}$.

Then the vector $u_{\delta}$ looks like

$u_{\delta}=\left(u_{1}, u_{2}, \ldots, u_{N_{u}}, \delta_{1} u, \delta_{2} u, \ldots, \delta_{N_{\delta}} u\right)^{T}$,

where $T$ is the matrix transposition operator. It is clear that equalities (2.3) and (2.6) are correct in this case. Physically, this means that $N_{\delta}$ measurements are false, and $N_{u}$ ones are precise. The results presented above testify to the following theorem.

$\mathrm{T} \mathrm{h}$ e o $\mathrm{r}$ e $\mathrm{m}$. Let we have a system of linear algebraic equations in the form (2.1). The validity of relation (2.3) means that the components of the vector $u_{\delta}=u+\delta u$ on the right-hand side of system (2.1) consist of either absolutely precise values $u$ or absolutely wrong values $\delta u$.

As Academician V.I. Arnold emphasized, "Mathematics, as well as physics, is an experimental science..." [23, p. 1323]. An experiment in mathematics consists of calculations. Therefore, let us numerically evaluate the quantities that enter equality (2.5) on the basis of the following examples.

Example 1. Let the mathematical model of a certain physical problem can be described by a SLAE with the right-hand side in the form

$f=\left(\sqrt{2 \pi}, \sqrt{3}-1, \pi^{2} / 4,5 / 3, e / 2, C\right.$,

$\left.\lg e, \ln \pi, \ln 10, e^{\pi / 2}\right)^{T}$,

whose dimension equals $N=10$. Let the values of the components of the vector $f$ be precise to six digits after the decimal point, i.e.

$u=(2.506628,0.732051,2.467402,1.666667$,

ISSN 2071-0194. Ukr. J. Phys. 2017. Vol. 62, No. 6
1.359141, 0.577216, 0.434294, 1.144730,

$2.302585,4.810477)^{T}$.

In this case,

$\pi=3.141593, \quad e=2.718282, \quad C=0.577216$.

At the same time, the values of the same components but with two digits after the decimal point are assumed to be approximate:

$u_{\delta}=(2.51,0.73,2.47,1.67,1.36,0.58,0.43$,

$1.14,2.30,4.81)^{T}$.

In this case,

$\delta u=\left(3.372 \times 10^{-3},-2.051 \times 10^{-3}, 2.598 \times 10^{-3}\right.$,

$3.333 \times 10^{-3}, 8.59 \times 10^{-4}, 2.784 \times 10^{-3}$,

$-4.294 \times 10^{-3},-4.730 \times 10^{-3},-2.585 \times 10^{-3}$,

$\left.-4.77 \times 10^{-4}\right)^{T}$

and

$$
\begin{aligned}
& (u, \delta u)=6.164484 \times 10^{-3}, \\
& \left(u_{\delta}, \delta u\right)=6.25413 \times 10^{-3}, \\
& \left(u_{\delta}, \delta u\right)-(u, \delta u)=\|\delta u\|^{2}=8.9646 \times 10^{-5}, \\
& (\delta u, \delta u)=\|\delta u\|^{2}=8.9645 \times 10^{-5} .
\end{aligned}
$$

Slightly changing the value of the first component of the vector $u_{\delta}$ by putting it equal to 2.50 , we obtain

$\tilde{u}_{\delta}=(2.50,0.73,2.47,1.67,1.36,0.58,0.43$,

$1.14,2.30,4.81)^{T}$,

$\delta \tilde{u}=\tilde{u}_{\delta}-u=\left(-6.628 \times 10^{-3},-2.051 \times 10^{-3}\right.$,

$2.598 \times 10^{-3}, 3.333 \times 10^{-3}, 8.59 \times 10^{-4}$,

$2.784 \times 10^{-3},-4.294 \times 10^{-3},-4.730 \times 10^{-3}$,

$\left.-2.585 \times 10^{-3},-4.77 \times 10^{-4}\right)^{T}$,

$(u, \delta \tilde{u})=-1.8901796 \times 10^{-2}$,

$\left(\tilde{u}_{\delta}, \delta \tilde{u}\right)=-1.877959 \times 10^{-2}$,

$\left(\tilde{u}_{\delta}, \delta \tilde{u}\right)-(u, \delta \tilde{u})=\|\delta \tilde{u}\|^{2}=1.22206 \times 10^{-4}$,

$(\delta \tilde{u}, \delta \tilde{u})=\|\delta \tilde{u}\|^{2}=1.22205 \times 10^{-4}$.

Example 2.

Let us evaluate the influence of a dimension of the vector on the right-hand side of SLAE (2.1) on the magnitude of the scalar product $(u, \delta u)$. Let a vector

483 
with the large dimension $F$ be formed by cyclically repeating the components of the vector

$f=\left(f_{i}\right)^{T}=\left(\sqrt{2 \pi}, \sqrt{3}-1, \pi^{2} / 4,5 / 3, e / 2, C\right.$, $\left.\lg e, \ln \pi, \ln 10, e^{\pi / 2}\right)^{T}$,

i.e. $F=\left(f_{i}, f_{i}, \ldots, f_{i}, \ldots, f_{i}\right)^{T}$. Let the cycle number equal $K$, so that $N=10 K$.

It is easy to see that, according to relations (2.9), at $u=F$, we have

$$
\begin{aligned}
& (u, \delta u)=K \times 6.164484 \times 10^{-3} \\
& \left(u_{\delta}, \delta u\right)=K \times 6.25413 \times 10^{-3} \\
& \left(u_{\delta}, \delta u\right)-(u, \delta u)=\|\delta u\|^{2}=K \times 8.9646 \times 10^{-5} .
\end{aligned}
$$

For instance, if $K=1000$, then $(u, \delta u)=6.164484$, i.e. the magnitude of the scalar product $(u, \delta u)$ increases with the growth of the dimension of the vector on the right-hand side of SLAE.

Now, following V.N. Strakhov, due to the CauchyBuniakowski inequality, we may write that

$(u, \delta u)^{2} \leqslant\|u\|^{2}\|\delta u\|^{2}$.

From whence, we obtain the estimate

$0 \leqslant \rho^{2} \leqslant 1$

where

$\rho^{2}=\frac{(u, \delta u)^{2}}{\|u\|^{2}\|\delta u\|^{2}}, \quad\|\delta u\|^{2} \neq 0$.

In the case $u=F$ for the quantities used in relation (2.14), we have

$(F, \delta F)^{2}=K^{2}(u, \delta u)^{2}$,

$\|F\|^{2}=K\|u\|^{2}, \quad\|\delta F\|^{2}=K\|\delta u\|^{2}$.

Hence, as one can see on the basis of equality (2.14), for the selected structure of the vector $F$, the quantity $\rho^{2}$ does not depend on its dimension.

Note that, for the $u$ - and $\delta u$-values used in Eq. (2.9), we have

$\rho^{2}=\frac{\left(6.164484 \times 10^{-3}\right)^{2}}{47.81 \times 8.9645 \times 10^{-5}}=0.008867$.

But if we use the corresponding values from Eq. (2.10), we obtain

$\rho^{2}=\frac{\left(-1.89 \times 10^{-2}\right)^{2}}{47.81 \times 1.22206 \times 10^{-4}}=0.06115$.

484
Therefore, a very small variation in the values of the quantities $\delta u$ used in examples (2.9) and (2.10) gives rise to the change of $\rho^{2}$ by an order of magnitude.

The examples above undoubtedly demonstrate that there is no orthogonality between the useful signal vectors $u$ and the error vectors $\delta u$. The postulation of the rigorous hypothesis $(u, \delta u)=0$ results only in that two systems of equations,

$A_{1} x_{1}=u$ and $A_{2} x_{2}=\delta u$,

rather than SLAE (2.1), have to be considered. Concerning an experiment, in which $N_{\delta}$ measurements are wrong and $N_{u}$ ones are absolutely precise, it can be characterized with the use of the expression "There is no better experiment that would have been worse than this one".

The contribution by Academician V.N. Strakhov to the development of the theory and practice of the solution of ill-posed problems is significant and wellknown (e.g., see the references in work [12]). However, his hypothesis (2.3) turned out, unfortunately, to be erroneous. It should be noted that the subject matter of this section is based on the content of work [24].

\section{Mathematical Interpretation of the Optical Experimental Data}

Unlike the interpretation variant where $(u, \delta u)=0$, which has a theoretical character and was considered in the previous section, we now discuss a possible plausible interpretation of the presence of errors in the experimental data of an optical experiment.

Conditionally, our example is associated with experimental researches of optical confinement effects in thin nanostructured films of various silicon carbide polytypes. This is a promising medium for applications under extreme conditions of high and low temperatures, under considerable radiation loadings, and in a chemically active environment $[25,26]$. The results of researches showed, in particular, that no optical confinement effect, neither at the main lasing wavelength of a neodymium laser $(\lambda=1064 \mathrm{~nm})$ nor at its second harmonic $(\lambda=532 \mathrm{~nm})$, was revealed in a silicon carbide specimen mainly consisting of an amorphous phase. A similar result was also obtained for a specimen consisting of the crystalline phase (3C) of nano-sized silicon carbide to an extent of almost $100 \%$ owing to its additional annealing (Fig. 1). From

ISSN 2071-0194. Ukr. J. Phys. 2017. Vol. 62, No. 6 
those results, it follows that the dependence of the intensity of radiation passed through the specimen on the intensity of incident radiation has an almost linear character for the amorphous and 100\%-crystalline specimens.

The aim of our further consideration is to reveal a plausible interpretation that does not strictly account for the errors in registered data. Therefore, we use other linear dependences of this kind, but with the number of experimental points larger by an order of magnitude and with more strongly pronounced measurement errors.

In Fig. 2, a typical dependence (experiment 1) of the relative magnitude of a registered signal (e.g., when a beam passes through a limiting diaphragm without a specimen) on the input signal is shown:

$u_{\delta}(x)=\frac{I_{\delta}^{(\mathrm{out})}}{I_{\max }}, \quad x=\frac{I^{(\mathrm{in})}}{I_{\max }}$.

A similar dependence (experiment 2) of the relative magnitude of the total signal transmitted through the specimen on the laser radiation intensity is depicted in Fig. 3:

$u_{\delta s}(x)=\frac{\tilde{I}_{\delta}^{\text {(out) }}}{I_{\max }}, \quad x=\frac{I^{(\text {in })}}{I_{\max }}$.

With a rather high confidence, we may assert that the both dependences have a linear character. Really, approximating the experimental data by linear functions, we obtain

$\hat{u}_{\delta}(x)=a_{1}+b_{1} x, a_{1}=0.00485266, b_{1}=1.22719 ;$

$\hat{u}_{\delta s}(x)=a_{2}+b_{2} x, a_{2}=-0.00175473, b_{2}=1.07823$.

The maximum approximation errors in the first and second cases are equal to $\delta \hat{u}=0.015$ and $\delta \hat{u}_{s}=0.012$, respectively. The results of approximations (3.1) and (3.2) are plotted in Fig. 3.

If the results of the experiment without a specimen (Fig. 2) are approximated by a cubic polynomial,

$$
\begin{aligned}
& \bar{u}_{\delta}(x)=a_{3}+b_{3} x+c_{3} x^{2}+d_{3} x^{3}, a_{3}=-0.0147916, \\
& b_{3}=1.53318, c_{3}=-1.16683, d_{3}=1.26578
\end{aligned}
$$

the obtained maximum approximation error equals $\delta \bar{u}=0.0145$, which is less than in the case of the linear approximation.

ISSN 2071-0194. Ukr. J. Phys. 2017. Vol. 62, No. 6

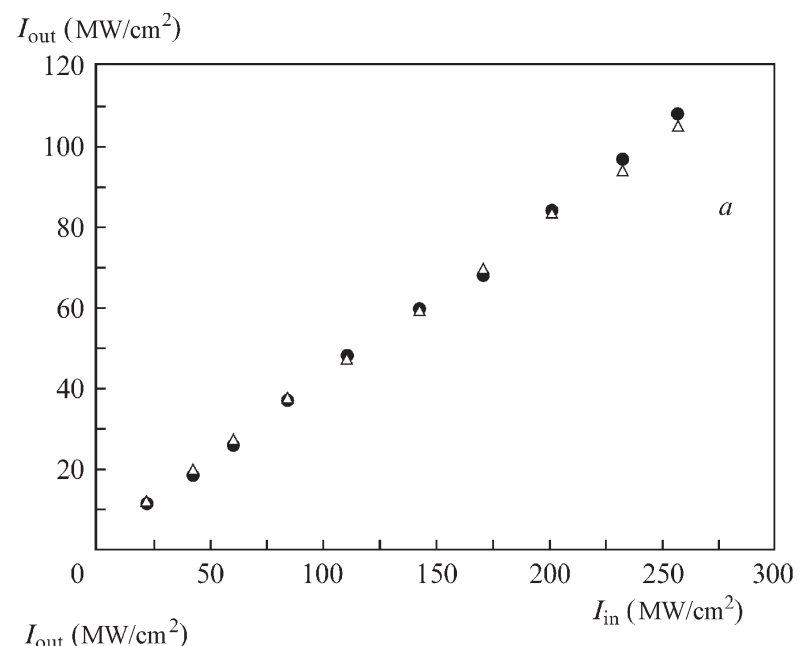

$I_{\text {out }}\left(\mathrm{MW} / \mathrm{cm}^{2}\right)$

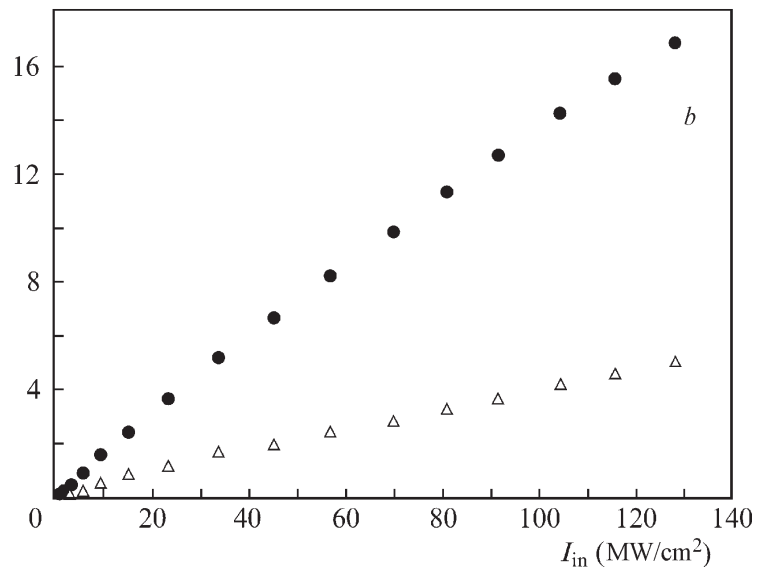

Fig. 1. Dependences of the radiation intensity passed through a specimen on the incident radiation intensity for the amorphous $(\bullet)$ and $100 \%$-crystalline $(\triangle, 3 C)$ specimens at $\lambda=1064$ (a) and $532 \mathrm{~nm}(b)$

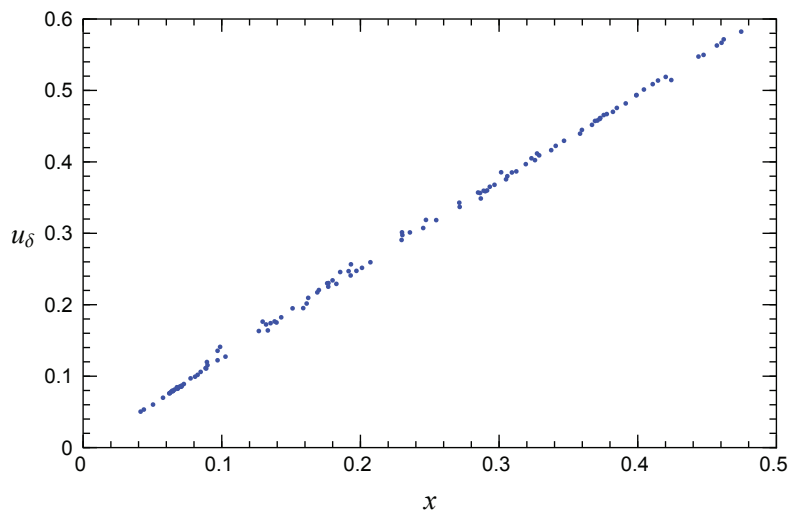

Fig. 2. Dependence of the relative magnitude of a registered signal on the input signal (experiment 1) 


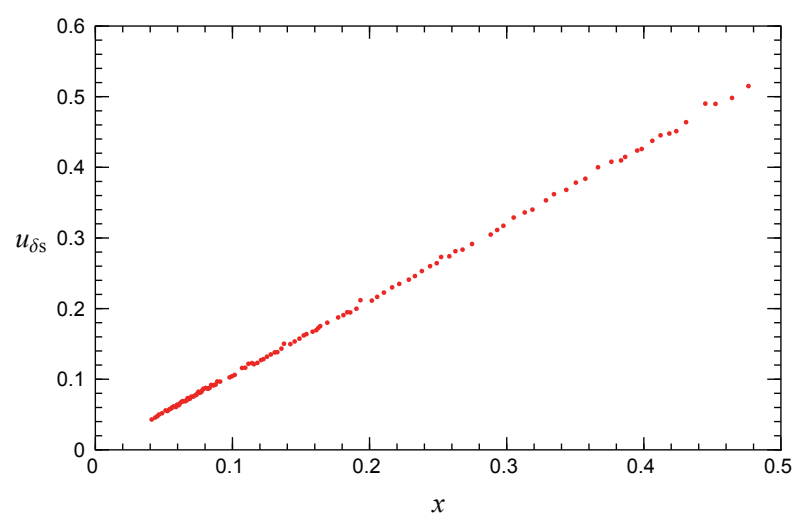

Fig. 3. Dependence of the relative magnitude of the total specimen transmission signal on the laser radiation intensity (experiment 2)

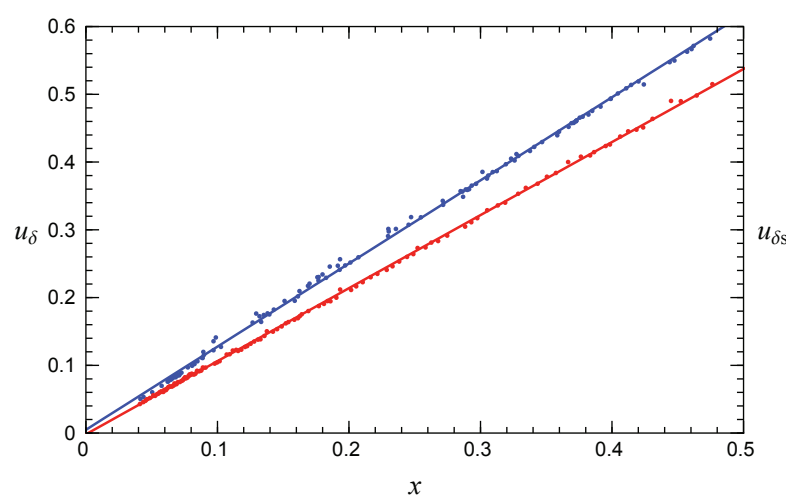

Fig. 4. Approximation of experimental data by linear functions

A principal meaning of the fact that the coefficients $a_{i} \quad(i=1,2,3)$ are nonzero for all approximations (3.1)-(3.3) should be pointed out. In other words, in the absence of an input signal, the measuring equipment registers the presence of a signal in the first and second experimental variants. This signal is nothing else but a simulation error at the coordinate origin,

$\delta u(0)=u_{\delta}(0)-u(0) \neq 0$.

Ignoring this fact may give rise to a false physical interpretation of the result of experimental researches. As a matter of fact, the measurement results obtained in the second experiment are quite often compared with the data of the first one by dividing those data by each other: $\frac{\hat{u}_{\delta s}(x)}{\hat{u}_{\delta}(x)}$. Such a procedure has a sense, e.g., in the case of a perfect measuring equipment that excludes any error. Provided

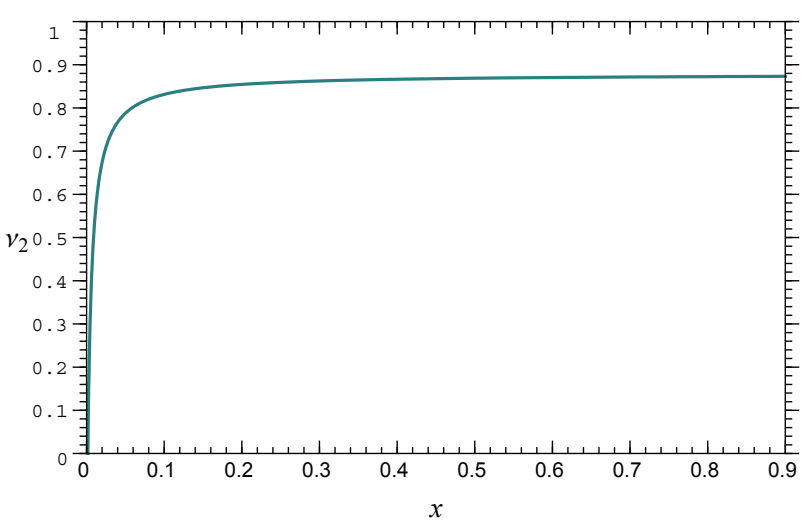

Fig. 5. Function $\nu_{1}(x)$ at $a_{1}=0$ in formula (3.1)

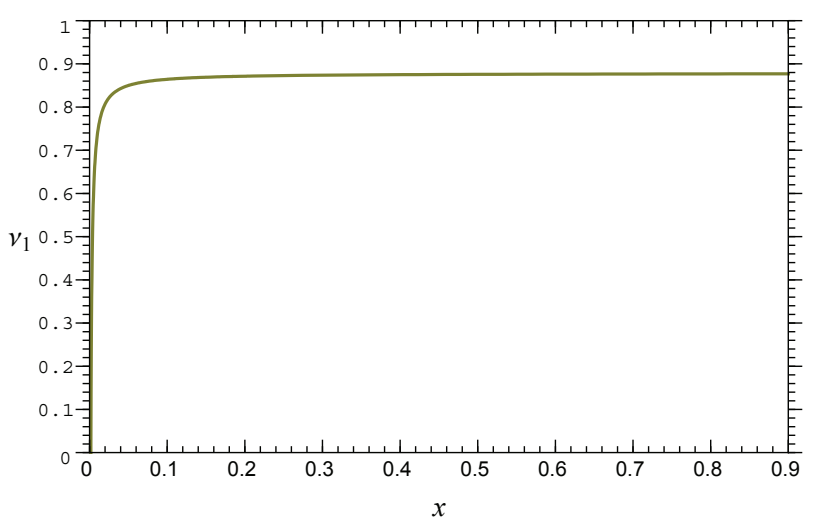

Fig. 6. Function $\nu_{1}(x)$ in the general case $\left(a_{1} \neq 0, a_{2} \neq 0\right)$

ideal conditions, all experimental points lie exactly on a straight line, which, in turn, passes through the coordinate origin.

Let us illustrate the aforesaid, by using simple calculations. Let $a_{1}=0$ and $a_{2}=0$. Then

$\frac{\hat{u}_{\delta s}(x)}{\hat{u}_{\delta}(x)}=\frac{\hat{b}_{2} x}{\hat{b}_{1} x}=\mathrm{const} \quad\left(\hat{b}_{1} \neq 0\right)$.

It is significant that either this is a perfect variant, or the experimental data are approximated by linear functions $u_{\delta i}(x)=b_{i} x$ in the course of their preliminary processing. It is clear that the matter concerns experiments, whose results are similar to those depicted in Figs. 2 and 3.

Let us consider a rather simple, but real case where the measurement data are accompanied by errors, but the straight line corresponding to the first measurements strictly crosses zero (i.e. $a_{1}=0$ ); in other words, there is no observable signal in the absence of 


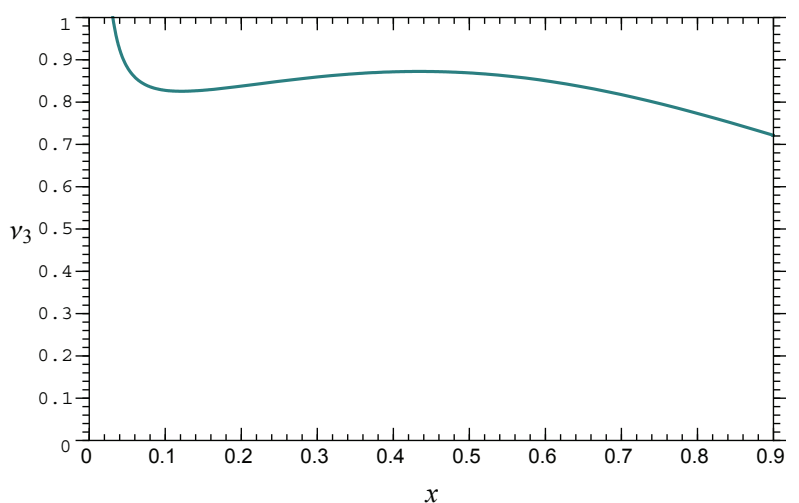

Fig. 7. Function $\nu_{3}(x)$

the input signal. Let all other coefficients have their previous values from experiments 1 and 2 . Then,

$\nu_{1}(x)=\frac{\hat{u}_{\delta s}(x)}{\tilde{u}_{\delta}(x)}=\frac{a_{2}+b_{2} x}{b_{1} x}=\frac{b_{2}}{b_{1}}+\frac{a_{2}}{b_{1} x}=\alpha_{1}+\frac{\beta_{1}}{x}$,

$\alpha_{1}=0.878614, \beta_{1}=-0.00142988$,

and the obtained curve is an ordinary hyperbole. In the case where all coefficients in Eqs. (3.1) and (3.2) are different from zero, a hyperbolic dependence is obtained as well (see Fig. 6).

Now, let us imagine an abstract experimenter. For some reasons, he disregards Eq. (3.4), being more interested in a variant where the results of the first experiment are approximated by the cubic polynomial (3.3). Moreover, the functions $\nu_{1}(x)$ and $\nu_{2}(x)$ behave unordinarily in the vicinity of zero. As a result, the function $\nu_{3}(x)$ obtains a graphic profile (see Fig. 7), which could invoke a desire to seek a certain "profound" physical sense.

As a matter of fact, if the function $\bar{u}_{\delta}(x)$ in formula (3.3) is factorized,

$\bar{u}_{\delta}(x)=(x-0.00971879) \times$

$\times\left(1.26578 x^{2}-1.15452 x+1.52195\right)$,

one can see that the value $x^{*}=0.00971879$ is a special point of the function

$\nu_{3}(x)=\frac{\hat{u}_{\delta s}(x)}{\bar{u}_{\delta}(x)}=$

$=\frac{1.07823 x-0.00175473}{(x-0.00971879)\left(1.26578 x^{2}-1.15452 x+1.52195\right)}$

and its derivatives. Hence, the pole singularity governs the character and the behavior of the considered function $\nu_{3}(x)$.
In order to avoid the incorrect interpretation of experimental results, we recommend to use the ratio between the derivatives of the functions, $\frac{\hat{u}_{\delta s}^{\prime}(x)}{\hat{u}_{\delta}^{\prime}(x)}=\frac{b_{2}}{b_{1}}$, rather than the ratio between the functions themselves, $\frac{\hat{u}_{\delta s}(x)}{\hat{u}_{\delta}(x)}$.

\section{Conclusions}

To summarize, using model examples for the results of scientific experimental researches, we showed the following.

1. Any hypotheses and assumptions concerning the level of measurement errors, their deterministic relation to predicted precise data have to be carefully and comprehensively analyzed in the combination with the results of computational experiments aimed at the solution of specific ill-posed problems. A necessary condition required for such an analysis to be complete is the comparison of the results of physical and computational experiments.

2. The polynomial approximation of the experimental dependences of an optical experiment (as is often done) together with inattentive attitude to the presence of errors in the experimental data (especially, in the vicinity of the coordinate origin) can result in a seemingly plausible, but incorrect physical interpretation of the obtained results.

1. W.K. Heisenberg. What is "apprehension" in theoretical physics. Priroda 4, 75 (1971) (in Russian).

2. A.S. Davydov. Interpretation of the results of scientific research in the physics domain. Preprint (Institute of Theoretical Physics, Kyiv, 1980) (in Russian).

3. A.N. Tikhonov, V.Ya. Arsenin. Solutions of Ill-Posed Problems (Wiley, 1977).

4. A. Bakushinsky, A. Goncharsky. Ill-Posed Problems: Theory and Applications (Kluwer, 1994).

5. G.M. Vainikko, A.Yu. Veretennikov. Iteration Procedures in Ill-Posed Problems (Nauka, 1986) (in Russian).

6. V.N. Strakhov. Critical analysis of the classical theory of linear ill-posed problems. Geofizika 3, 3 (1999) (in Russian).

7. V.N. Strakhov. Geophysical "Dialect" of Mathematic Language (OIFZ RAN, 2000) (in Russian).

8. V.N. Strakhov. On the Training of Specialists in the Field of Gravity Prospecting and Magnet Prospecting at Higher Educational Institutions of Russia (OIFZ RAS, 2000) (in Russian).

9. V.N. Strakhov. Geophysical version of the theory of regularization of linear ill-posed problems. In Problems of the Theory and Practice of the Geological Interpretation of 
Gravitational, Magnetic, and Electric Fields (Institute of Geophysics of NASU, OIFZ RAS, 2001), p. 99 (in Russian).

10. V.N. Strakhov. Basic constructive ideas used at the development of numerical methods for finding stable approximate solutions of the systems of linear algebraic equations with an approximately given right-hand side, which arise in the gravimetry and magnetometry problems. I. Constructive ideas of the fundamental level. Fizika Zemli 11, 3 (2001) (in Russian).

11. V.N. Strakhov. On the problem of regularization of the systems of linear algebraic equations that arise in the gravimetry and magnetometry problems. I. New constructive ideas. In Actual Problems of Mathematical Geophysics (OIFZ RAN, 2001), Vol. 1, p. 69 (in Russian).

12. V.N. Strakhov. Paradigm Change in the Theory of Linear Ill-Posed Problems (OIFZ RAN, 2001) (in Russian).

13. V.N. Strakhov, A.V. Strakhov. On regularization of the least-squares method. Geofiz. Zh. 20, N 6, 18 (1998) (in Russian).

14. V.N. Strakhov, A.V. Strakhov. Generalization of the leastsquares method and regularized algorithms for finding stable approximate solutions of the systems of linear algebraic equations with approximate data that arise, while solving geophysical problems. Geofiz. Zh. 21, N 2, 3 (1999) (in Russian).

15. V.N. Strakhov. Generalization of M.A. Lavrent'ev and A.N. Tikhonov variational methods for the regularization of the systems of linear algebraic equations with an approximately given right-hand side, which provides the requirements of gravimetry and magnetometry. Geofiz. Zh. 24, N 5, 3 (2002) (in Russian).

16. V.N. Strakhov, A.V. Strakhov. Universal algorithms for the regularization of the systems of linear algebraic equations with an additive interference on the right-hand side, which arise at the solution of the gravimetry and magnetometry problems. Fizika Zemli 10, 3 (2000) (in Russian).

17. Q. Yang, J.T. Seo, S.J. Creekmore, D.A. Temple, K.P. Yoo, S.Y. Kim, S.S. Jung, A.G. Mott. I-scan measurements of the nonlinear refraction and nonlinear absorption coefficients of some nanomaterials. In Proc. SPIE 4797, 101 (2003) [DOI: 10.1117/12.453537].

18. I. Dancus, V.I. Vlad, A. Petris, T.B. Rujoiu, I. Rau, F. Kajzar, A. Meghea, A. Tane. Z-Scan and I-Scan methods for characterization of DNA optical nonlinearities. Rom. Rep. Phys. 65, 966 (2013).

19. G. Boudebs, K. Fedus. Image formation and applications to third order nonlinear optical measurement. In Proceed- ings of the 14th International Conference on Transparent Optical Networks (ICTON) (2012), p. 1 [DOI: 10.1109/ ICTON.2012.6253855].

20. R.F. Souza, M.A. Alencar, J.M. Hickmann, R. Kobayashi, L.R. Kassab. Femtosecond nonlinear optical properties of tellurite glasses. Appl. Phys. Lett. 89, 171917 (2006) [DOI: 10.1063/1.2364467].

21. T.B. Rujoiu, A. Petris, V.I. Vlad. Nonlinear refractive properties of $1 \mathrm{D}$ periodically nanostructured silicon-on-insulator investigated by reflection I-Scan. In Proc. SPIE 8882, 88820C (2013) [DOI: 10.1117/12.2032351].

22. T.B. Rujoiu, V.I. Vlad, A. Petris. Measurement of nonlinear refractive index of silicon nanostructures using reflection intensity scan method. Rom. Rep. Phys. 65, 954 (2013).

23. V.I. Arnold. Mathematics and physics: a parent and a child or sisters? Usp. Fiz. Nauk 169, 1311 (1999) (in Russian).

24. V.I. Starostenko, V.N. Starkov. On the results of verification of V.N. Strakhov hypothesis. Geofiz. Zh. 25, No. 1, 122 (2003) (in Russian).

25. A.A. Borshch, V.N. Starkov, V.I. Volkov, V.I. Rudenko, A.Yu. Boyarchuk, A.V. Semenov. Optical confinement effects in thin nanostructured silicon carbide films. Kvant. Elektron. 43, 1122 (2013) (in Russian).

26. A.A. Borshch, M.S. Brodyn, V.N. Starkov, V.I. Rudenko, V.I. Volkov, A.Yu. Boyarchuk, A.V. Semenov. Broadband optical limiting in thin nanostructured silicon carbide films and its nature. Opt. Commun. 364, 88 (2016) [DOI: 10.1016/j.optcom.2015.11.040].

Received 31.01.17.

Translated from Ukrainian by O.I. Voitenko

В.М. Старков, А.О. Бори, І.С. Ганджа, П.М. Томчук

ПРИКЛАДИ ПРАВДОПОДІБНОЇ

ІНТЕРПРЕТАЦЇ̈ РЕЗУЛЬТАТІВ

ЕКСПЕРИМЕНТАЛЬНИХ ДОСЛІДЖЕНЬ

Р е $з$ ю м е

На основі строгої математичної теорії інтерпретації експериментальних даних розглянуті приклади правдоподібної (але невірної) інтерпретації остаточних експериментальних досліджень. Показано, що апаратні похибки можуть суттєво впливати на результати вимірювань величин оптичного експерименту. Проведений нами аналіз доводить, що ігнорування цього факту може призвести до помилкових висновків щодо фізичної суті розглянутих оптичних явищ. 